\title{
MODELAGEM DA POLIMERIZAÇÃO DO ÁCIDO ACRÍLICO
}

\author{
J. C. Coimbra ${ }^{1 *}$, A. L. T. Brandão ${ }^{2}$, B. F. Oechsler ${ }^{3}$, M. C. C. Pinto ${ }^{4}$, J. C. Pinto ${ }^{5}$, P. A. \\ Melo $^{5}$ \\ ${ }^{1}$ Universidade Federal do Rio de Janeiro, Programa de Engenharia Química \\ E-mail para contato: jamille@ peq.coppe.ufrj.br
}

RESUMO - Um modelo matemático para polimerização do ácido acrílico (AA) em um processo batelada é proposto neste trabalho. A partir deste, foi possível simular o avanço da massa molar média ponderal, e da distribuição de massas molares. Além disso, foi possível estimar parâmetros cinéticos,não disponíveis na literatura,desse sistema reacional. Os resultados obtidos pela modelagem foram validados satisfatoriamente com dados experimentais obtidos em um reator em escala de bancada.

\section{INTRODUÇÃO}

O poli(ácido acrílico) (PAA) é um polímero amplamente utilizado na produção de resinas para uso geral bem como aplicações em ortopedia dentária(ARTURSSON, 1994). As propriedades deste polímero dependem fortemente da combinação de reações químicas que afetam o processo de produção. Sua qualidade é também dependente das condições de operação que são cruciais para a produção eficiente e garantia da qualidade do produto formado.Neste contexto, a elaboração de um modelo matemático pode ser útil para o melhor entendimento das dificuldades inerentes da polimerização deste sistema desde que seja ancorado em um mecanismo cinético baseado em evidência experimental. Nesse contexto, destacam-se os trabalhos de ZALDÍVAR et al. (1997) e MINARI et al. (2011). O primeiro trabalho aborda a modelagem matemática da copolimerização do AA com acetato de vinila e o segundo avalia a influência das insaturações do AA na cinética da reação e, consequentemente, no processo de produção deste polímero. Este trabalho pôde reproduzir por simulação computacional a distribuição das massas molares do PAA sob a ótica não reportada na literatura para este processo.

Os objetivos deste trabalho são (i) elaborar um modelo que descreva a evolução das distribuições de massas molares do polímero formado bem como suas médias (ii) estimar parâmetros cinéticos do modelo e (iii) validar o modelo a partir dos dados experimentais obtidos em um reator em escala de bancada.

\section{MODELAGEM MATEMÁTICA}

\subsection{Mecanismo Cinético}

A cinética de polimerização do ácido acrílico em solução aquosa é composta por três etapas principais: iniciação, propagação e terminação. Há também a presença de mecanismos de transferência de cadeia que provocam uma diminuição das massas 
molares das cadeias poliméricas (Tabela 1). Para a proposta da modelagem cinética, é importante que se estabeleçam as hipóteses consideradas, a saber: hipótese da cadeia longa (ou seja, todas as cadeias apresentam a mesma velocidade de reação e os termos de consumo de monômero são desprezíveis, frente a propagação); hipótese do estado quase-estacionário para os radicais (essas espécies são altamente reativas e, por isso,considera-se que não existe acúmulo no meio reacional); não foi considerada a etapa de terminação por combinação, mas apenas por desproporcionamento, uma vez que a viscosidade do meio é elevada (ODIAN, 2004).A abordagem de balanços populacionais foi aplicada ao mecanismo cinético de polimerização via radicais livres, a fim de obter os balanços molares das espécies poliméricas envolvidas no meio reacional.

Tabela 1: Etapas da reação de polimerização do PAA.

\begin{tabular}{l|c}
\hline \multicolumn{1}{c}{ Etapa do Mecanismo } & Reação \\
\hline Iniciação & $I \stackrel{k d}{\longrightarrow} 2 R^{\bullet}$ \\
Propagação & $R^{\bullet}+M \stackrel{k_{1}}{\longrightarrow} P_{1}$ \\
Transferência para o monômero & $P_{i}+M \stackrel{k p}{\longrightarrow} P_{i+1}$ \\
& $P_{i}+M \stackrel{k t m}{\longrightarrow} D_{i}+P_{1}$ \\
Terminação por desproporcionamento & $P_{i}+P_{j} \stackrel{k t d}{\longrightarrow} D_{i}+D_{j}$ \\
Transferência para os agentes de transferência & $P_{i}+C T A_{1} \stackrel{k t r c t a_{1}}{\longrightarrow} D_{i}+C T A_{1}^{\bullet}$ \\
decadeia(CTA): & $C T A_{1}^{\bullet}+M \stackrel{k i c t a_{1}}{\longrightarrow} P_{1}$ \\
$C T A_{1}$ - etanol & $P_{i}+C T A_{2} \stackrel{k t r c t a_{2}}{\longrightarrow} D_{i}+C T A_{2}^{\bullet}$ \\
$C T A_{2}$ - ácido itacônico & $C T A_{2}^{\bullet}+M \stackrel{k i c t a a_{2}}{\longrightarrow} P_{1}$ \\
\hline
\end{tabular}

As equações de balanços materiais de cada espécie presente no meio reacional são apresentadas abaixo e as variáveis respostas do modelo que se pretende analisar no trabalho são descritas conforme as Equações (8)-(10).

$$
\begin{aligned}
& \frac{d I}{d t}=-k d \frac{I}{V} V \\
& \frac{d M}{d t}=\left(-2 f k d \frac{I}{V}-k p \frac{M}{V} \frac{\mu_{0}}{V}-k t m \frac{M}{V} \frac{\mu_{0}}{V}-k_{\text {trctal }} \frac{\mu_{0}}{V} \frac{C T A_{1}}{V}-k_{\text {trcta2 }} \frac{\mu_{0}}{V} \frac{C T A_{2}}{V}\right) V \\
& \frac{d C T A_{1}}{d t}=\left(k t r_{C T A 1} \frac{C T A_{1}}{V} \frac{\mu_{0}}{V}\right) V \\
& \frac{d C T A_{2}}{d t}=\left(k t r_{\text {CTA2 }} \frac{C T A_{2}}{V} \frac{\mu_{0}}{V}\right) V \\
& \frac{d \lambda_{k}}{d t}=\left(k t m \frac{M}{V} \frac{\mu_{k}}{V}+k t \frac{\mu_{k}}{V} \frac{\mu_{0}}{V}+k t r_{\text {cta1 }} \frac{\mu_{k}}{V} \frac{C T A_{1}}{V}+k t r_{\text {cta } 2} \frac{\mu_{k}}{V} \frac{C T A_{2}}{V}\right) V
\end{aligned}
$$


$\frac{d \mu_{k}}{d t}=\left(\begin{array}{l}-2 f k d \cdot \frac{I}{V}-k p \frac{M}{V} \frac{\mu_{k}}{V}+k p \frac{M}{V} \sum_{i=1}^{\infty}(i+1)^{k} \frac{P_{i}}{V}-k t \frac{\mu_{k}}{V} \frac{\mu_{0}}{V}-k t r_{c t a 1} \frac{\mu_{k}}{V} \frac{C T A_{1}}{V} \\ +k t r_{c t a 1} \frac{\mu_{0}}{V} \frac{C T A_{1}}{V}-k t r_{c t a 2} \frac{\mu_{k}}{V} \frac{C T A_{2}}{V}+k t r_{c t a 2} \frac{\mu_{0}}{V} \frac{C T A_{2}}{V}+k t m \frac{M}{V} \frac{\mu_{0}}{V}-k t m \frac{M}{V} \frac{\mu_{k}}{V}\end{array}\right) V$

onde:

$V=\left(\frac{M_{m} M M_{m}}{\rho_{m}}+\frac{M M_{m}\left(\lambda_{1}+\mu_{1}\right)}{\rho_{P}}+\frac{M_{S} M M_{S}}{\rho_{S}}+\frac{M_{c t a 1} M M_{c t a 1}}{\rho_{c t a 1}}+\frac{M_{c t a 2} M M_{c t a 2}}{\rho_{c t a 2}}\right)$

$M n=\left(\frac{\mu_{1}}{\mu_{0}}\right) M M_{m}$

$M w=\left(\frac{\mu_{2}}{\mu_{1}}\right) M M_{m}$

$I P D=\frac{M_{w}}{M_{n}}$

\section{METODOLOGIA}

O estudo foi dividido em duas partes. Inicialmente, um plano experimental foi proposto e os dados experimentais foram coletados para o sistema de polimerização, composto por ácido acrílico (monômero), persulfato de potássio (iniciador), água (solvente) e isopropanol (agente de transferência de cadeia). A partir dessas informações, o erro experimental foi calculado com base nos experimentos eem pseudoréplicas, geradas a partir destes, por meio de uma perturbação aleatória dos dados apresentados na Tabela 2, abaixo. Para geração das pseudo-réplicas, admite-se que os erros seguem a distribuição normal, com desvio padrão igual a $10 \%$ do valor fornecido e média nula. As reações de polimerização foram realizadas durante três horas com diferentes concentrações de ácido acrílico e de persulfato de potássio (Tabela 2), a uma temperatura de $95^{\circ} \mathrm{C}$ no sistema de reação.

Tabela 2: Dados experimentais da reação de polimerização do ácido acrílico.

\begin{tabular}{ccccccccc}
\hline Amostra & $\mathrm{P}(\%)$ & $\mathrm{I}(\%)$ & $\mathrm{Ag}(\%)$ & $\mathrm{A}(\%)$ & $\mathrm{M}(\%)$ & Conversão & $\mathrm{Mw}(\mathrm{g} / \mathrm{mol})$ & $\mathrm{IP}$ \\
\hline 1 & 5,00 & 0,50 & 44,50 & 10,00 & 40,00 & 1,00 & $2,08 \times 10^{5}$ & 1,81 \\
2 & 5,00 & 0,50 & 54,50 & 8,00 & 32,00 & 1,00 & $1,84 \times 10^{5}$ & 1,79 \\
3 & 5,00 & 0,50 & 64,50 & 6,00 & 24,00 & 0,62 & $1,55 \times 10^{5}$ & 1,65 \\
4 & 5,00 & 0,50 & 44,50 & 10,00 & 35,00 & 1,00 & $2,19 \times 10^{5}$ & 1,88 \\
\hline
\end{tabular}

$\mathrm{P}$ - isopropanol, I - iniciador, $\mathrm{Ag}$ - água, $\mathrm{A}$ - ácido itacônico, $\mathrm{M}$ - monômero, $\mathrm{Mw}$ - massa molar média ponderal, IP - índice de polidispersão.

A segunda parte do estudo consistiu na modelagem da conversão do monômero edas distribuições de massas molares. Para problemas lineares de polimerização, o método dos momentos, em sua forma clássica,foi usado para fornecer as massas molares médiasdo polímero formado (MEYER et al.,2005). Além dos valores médios, 
as distribuições de massas molares também foram calculadas através do cômputo de momentos adicionais segundo uma técnica especial descrita em NELE (1999) e comparadas experimentalmente com as curvas fornecidas pelo equipamento de cromatografia por permeação em gel (GPC). Nessa técnica, a distribuição de Flory é usada como referência. O sistema de equações diferenciais foi resolvido com o integrador numérico DASSL (PETZOLD, 1989). Além disso, alguns parâmetros cinéticos desconhecidos foram estimados a partir dos dados experimentais das massas molares médias e do índice de polidispersão. Utilizou-se uma abordagem híbrida de estimação de parâmetros, baseada no método do enxame de partículas acoplado ao método de Gauss-Newton, implementado no pacote computacional Estima (SCHWAABet al., 2008).

\section{RESULTADOS E DISCUSSÃO}

No início da polimerização, a reatividade do monômero é alta, assim a reação se propaga intensamente, ocasionando maior valor de massa molar média ponderal (Mw). A partir de um determinado tempo da reação, as taxas de transferênciacomeçam a aumentar e as cadeias poliméricas crescem a uma taxa menor, terminação mais acentuada do polímero. Dessa forma, verifica-se que o valor de Mwé menor quando uma maior quantidade de agente de transferência foi empregada (Figura 1). Constata-se, portanto, que os agentes de transferência de cadeia isopropanol e ácido itacônico foram efetivos.Foi possível construir a curva de distribuição de massas molares a partir do modelo em questão, reproduzindo dados reais do processo de polimerização (Figura 2).

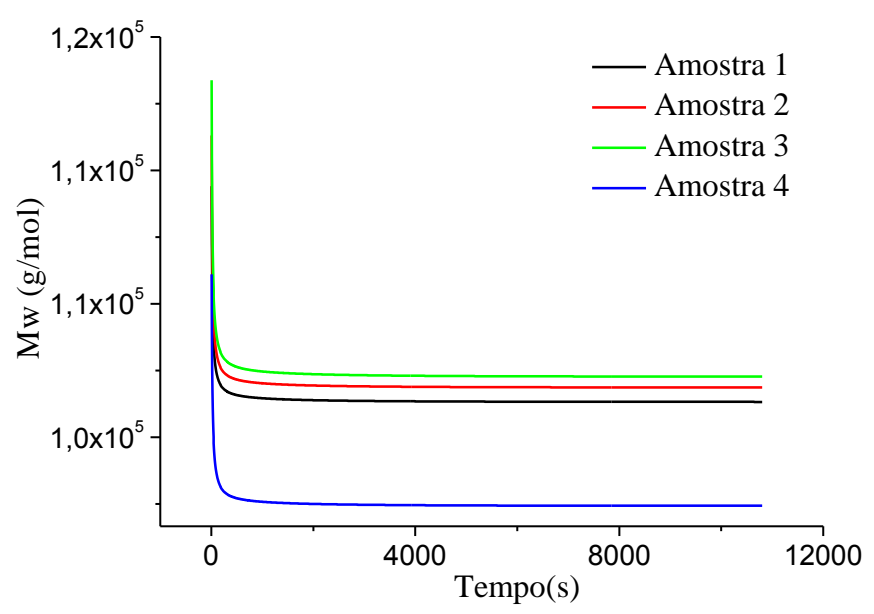

Figura 1: Simulações da massa molar média ponderal (Mw)ao longo de batelada.

As constantes cinéticas foram estimadas a partir do método não determinístico enxame de partículas, como discutido acima. Os resultados podem ser verificados na Tabela 3. Sabe-se que, quanto maior a amplitude do intervalo de confiança dos parâmetros, maior grau de incerteza pode estar associada à sua predição (SCHWAAB et al., 2007). Neste caso, pode-se dizer que o primeiro parâmetro apresentou uma estimação com menor incerteza, apesar disso, quando se considera a ordem de grandeza destes parâmetros, verifica-se que o intervalo de confiança é relativamente pequeno. 
A estimativa dos parâmetros baseou-se na minimização de uma função objetivo do tipo mínimos quadrados ponderados. Uma análise posterior indicou a correlação entre os parâmetros cinéticos estimados é de 0,478 , que pode ser considerada baixa. Este resultado pode ser satisfatoriamente interpretado como a baixa influência que uma variável exerce na outra. Neste sentido, foi possível perceber a importância dos dois agentes de transferência de cadeia que, embora exercendo o mesmo papel, isto é, interromper o sítio ativo da cadeia polimérica, estes operam em conjunto. Assim, é provável que um não interfira, significativamente, na atuação do outro.
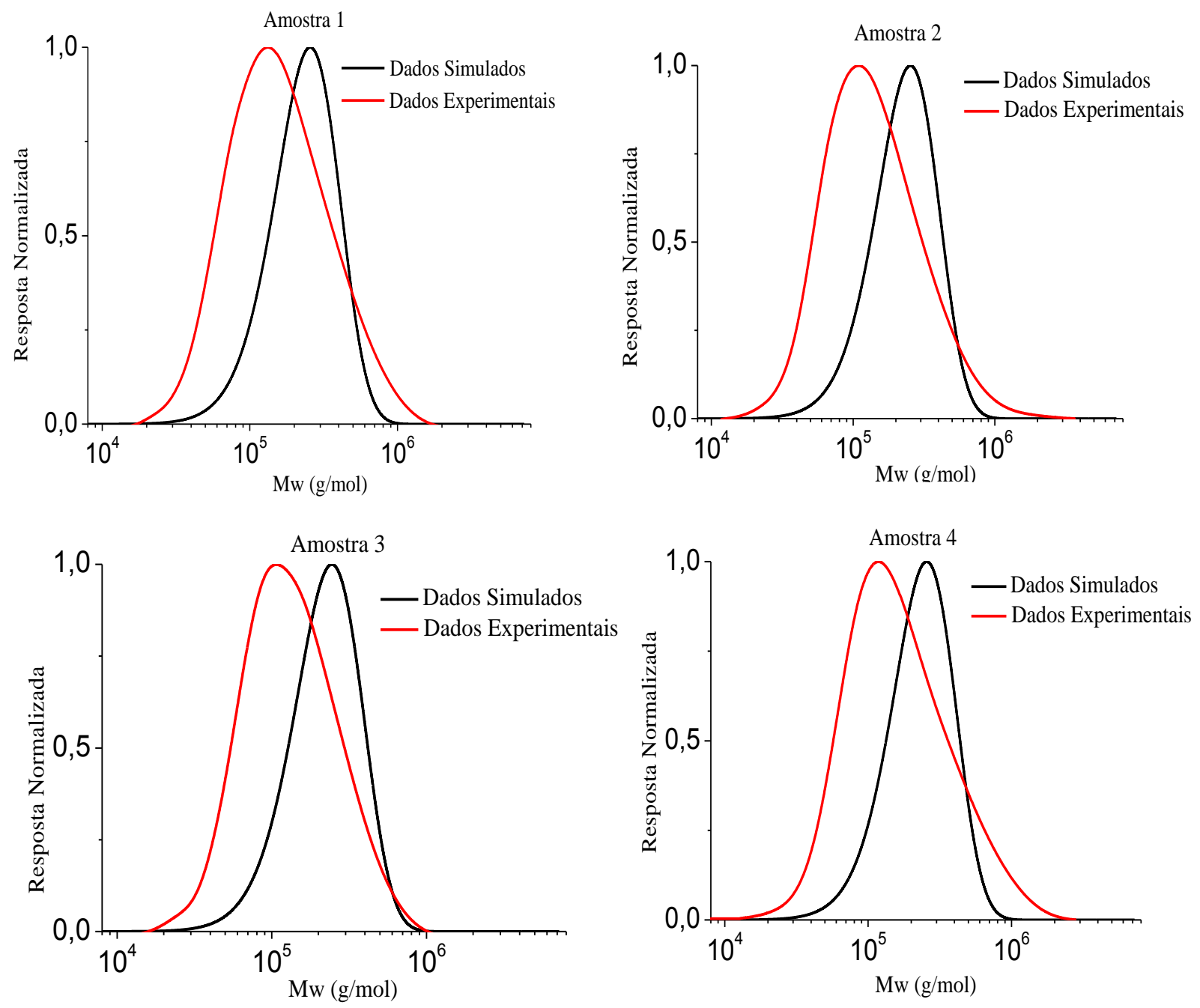

Figura 2: Distribuição de massa molar (Mw) versus resposta normalizada no final das amostras analisadas.

Tabela 3:Parâmetros estimados e respectivos intervalos de confiança.

\begin{tabular}{cccc}
\hline Parâmetro & Valor Estimado (L/mols) & Limite Inferior (L/mols) & Limite Superior(L/mols) \\
\hline trcta $_{1}$ & 48,865 & 48,195 & 49,431 \\
ktrcta $_{2}$ & 855,067 & 820,352 & 889,201 \\
\hline
\end{tabular}

ktrcta :Constante de transferência de cadeia, $k$ trcta $_{1}$ : etanol, $k$ trcta $_{2}$ : ácido itacônico 
A partir dos valores de massa molar média ponderal $(\mathrm{Mw})$ e do índice de polidispersão (IP) observados experimentalmente e preditos pelo modelo, é possível determinar suas respectivas incertezas (Figura 3). Verificou-se que, experimentalmente, não foi possível discriminar as amostras, uma vez que os erros experimentais informados na barra horizontal da Figura 3 se interceptam, resultando em alto grau de incerteza tanto para IP quanto para Mw. Diferentemente, o grau de incerteza do modelo matemático, dado pela barra vertical de erros, foi um pouco inferior, sendo possível fazer discriminação entre algumas amostras para os dois casos (SCHWAABet al., 2007).
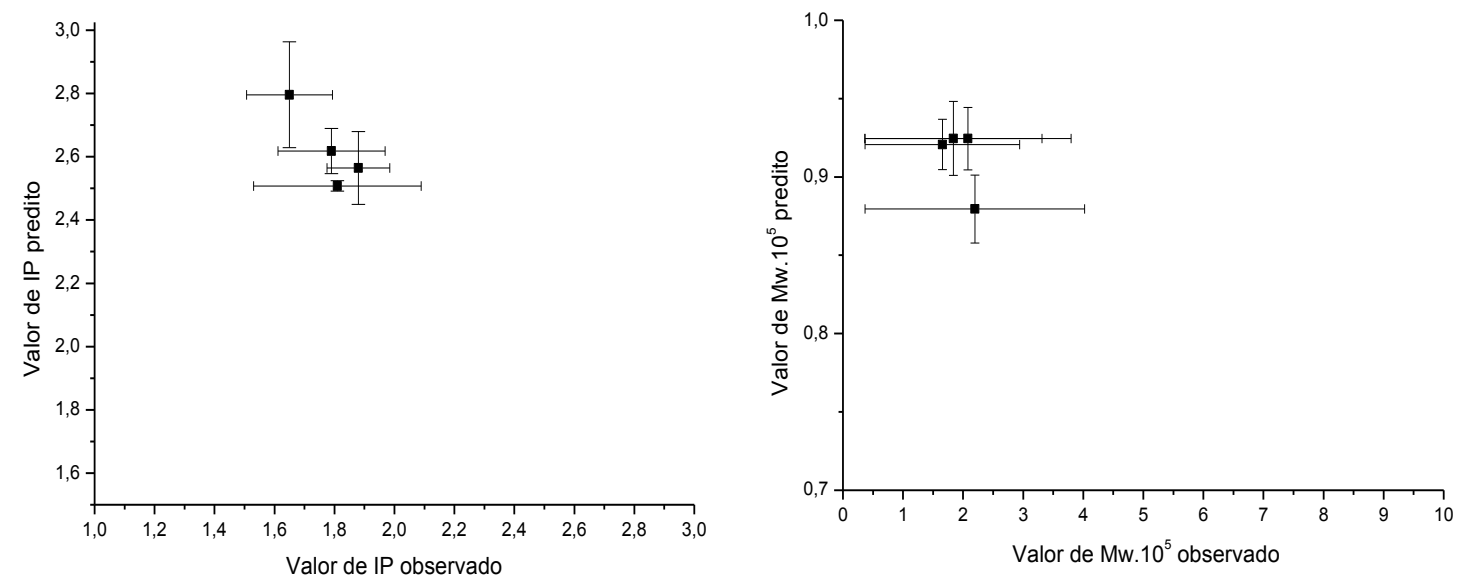

Figura 3:Valores preditos e observados experimentalmente para o índice de polidispersão (IP) e para a massa molar média ponderal (Mw).

\section{CONCLUSÕES}

Os resultados indicaram que um modelo cinético simplificado, com seis parâmetros, foi capaz de descrever bem o comportamento da massa molar média e do índice de polidispersão da polimerização do ácido acrílico. Além disso, foi possível reproduzir, por simulação computacional, a distribuição de massas molares obtida por cromatografia por permeação em gel (GPC). Após a estimaçãodos parâmetros cinéticos, o modelo foi validado experimentalmente com dados reais. Este sistema de polimerização auxilia na compreensão dahomopolimerização do ácido acrílico.

\section{NOMENCLATURA}

$k d$ : constante cinética de decomposição (1/s)

$k_{1}$ : constante cinética de iniciação (L/mol.s)

$k p$ : constante cinética de propagação (L/mol.s)

$\mathrm{ktm}$ : constante cinética de transferência para o monômero $(\mathrm{L} / \mathrm{mol} . \mathrm{s})$

$k t d$ : constante cinética de terminação por desproporcionamento (L/mol.s)

$k t c$ : constante cinética de terminação por combinação (L/mol.s)

$k t r c t a$ : constante cinética de transferência para o agente CTA (L/mol.s) 
kicta: constante cinética de transferência do agente CTA para o monômero (L/mol.s)

$I$ : iniciador (persulfato de potássio)

$R^{\bullet}$ : radical

$M$ :monômero (ácido acrílico)

$P_{i}$ : polímero vivo com tamanho de cadeia $i$

$D_{i}$ : polímero morto com tamanho de cadeia $i$

$C T A$ :agente de transferência de cadeia

$V$ :volume (L)

$f$ :fator de eficiência da iniciação

$\mu_{k}: \mathrm{k}^{\text {th }}$ momento da distribuição dos radicais poliméricos vivos

$\lambda_{k}: \mathrm{k}^{\text {th }}$ momento da distribuição dos radicais poliméricos mortos

$M_{m}$ : massa de monômero (g)

$M M_{m}$ : massa molar de monômero $(\mathrm{g} / \mathrm{mol})$

$M_{s}$ : massa de solvente $(\mathrm{g})$

$M M_{s}$ : massa molar de solvente $(\mathrm{g} / \mathrm{mol})$

$M_{c t a}:$ massa de agente de transferência $(\mathrm{g})$

$M M_{c t a}$ :massa molar de agente de transferência $(\mathrm{g} / \mathrm{mol})$

$\rho_{M}:$ densidade do monômero $(\mathrm{g} / \mathrm{L})$

$\rho_{P}$ :densidade do polímero $(\mathrm{g} / \mathrm{L})$

$\rho_{S}:$ densidade do solvente $(\mathrm{g} / \mathrm{L})$

$\rho_{c t a}$ :densidade do agente de transferência de cadeia $(\mathrm{g} / \mathrm{L})$

conv. : conversão

Press $_{i}$ : pressão parcial do componente i (Pa)

Press $_{i_{s a t}}$ :pressão de saturação do componente i (Pa)

$\phi_{v}^{i}$ : fração volumétrica do componente $i$

$\chi$ : parâmetro de Flory Huggins

Pr ess : pressão total $(\mathrm{Pa})$

Pr ess $_{\text {solvente }}$ :pressão do solvente no reator $(\mathrm{Pa})$

$M_{n}$ :massa molar média aritmética $(\mathrm{g} / \mathrm{mol})$

$M_{w}$ :massa molar média ponderal $(\mathrm{g} / \mathrm{mol})$

$I P D$ : índice de polidispersão

\section{REFERÊNCIAS}

ARTURSSON, P. et al. Chitosan on the permeability of monolayers of intestinal epithelial cells (caco-2). Pharm. Research, v. 11. P. 1358-1361. 1994.

MEYER, T.; KEURENTJES, J. Handbook of Polymer Reaction Engineering.Weinheim: Wiley, 2005. p.249-322. 
MINARI, R. J, et al. Semibach Aqueous- Solution Polymerization of Acrylic Acid:

Simultaneous Control of Molar Masses and Reaction Temperature.

Macromol.React.Eng, v.5, p. 223-231, 2011.

NELE M., SAYER C., PINTO J.C., 1999.“Computation of molecular weight distributions by polynomial approximation with complete adaptation procedures", Macromol.Theory Simul. v. 8, n. 3, p. 199-213.

ODIAN, G., Principles of Polymerization, 4 ed. New Jersey, John Wiley and Sons Inc., 2004.

PETZOLD, L. R., 1989. DASSL code, version 1989, Computing and Mathematics Research Division, Lawrence Livermore National Laboratory, L316, Po Box 808, Livermore, CA 94559.

SCHWAAB, M., PINTO, J. C., 2007, Análise de Dados Experimentais I Fundamentos de Estatística e Estimação de Parâmetros, e-papers, Rio de Janeiro.

SCHWAAB, M., BISCAIA Jr., E. C., MONTEIRO, J. L., PINTO, J. C., 2008, "Nonlinear Parameter Estimation through Particle Swarm Optimization", Chemical Engineering Science, v. 63, pp. 1542-1552.

SILVA, F.M. et al. Acrylic/Vinyl Acetate Suspension Copolymerizations. 2. Modeling and Experimental Results. Ind. Eng. Chem. Res, v.43, p 7324-7342, 2004.

ZALDÍVAR et al. One the Preparation of Acrylic Acid/vinyl Acetate Copolymers with Constant Composition:4. Modelling Batch and Continuous Free-Radical AA/VA Copolymerization.Elsevier Science, v. 38, p. 5823-5833, 1997. 
ANEXO

Emresposta a dúvida do revisor de que a queda de Mw nosinstantesiniciaisseriadevidoaoefeito da inicialização do programaou da variação da temperatura do meioreacional, afirmoserdevidoaoefeito da inicialização do programa. 